\title{
Marketing expérientiel et hyperréalité dans le domaine du tourisme culturel
}

Experiential marketing and hyperreality in the field of cultural tourism

Dominique Bourgeon-Renault et Elodie Jarrier

\section{OpenEdition}

\section{Journals}

Édition électronique

URL : http://journals.openedition.org/tourisme/1623

DOI : 10.4000/tourisme.1623

ISSN : 2492-7503

Éditeur

Éditions touristiques européennes

Référence électronique

Dominique Bourgeon-Renault et Elodie Jarrier, « Marketing expérientiel et hyperréalité dans le domaine du tourisme culturel », Mondes du Tourisme [En ligne], 14 | 2018, mis en ligne le 30 juin 2018, consulté le 19 avril 2019. URL : http://journals.openedition.org/tourisme/1623 ; DOI : 10.4000/ tourisme.1623

Ce document a été généré automatiquement le 19 avril 2019.

Mondes du tourisme est mis à disposition selon les termes de la licence Creative Commons Attribution - Pas d'Utilisation Commerciale - Pas de Modification 4.0 International. 


\title{
Marketing expérientiel et hyperréalité dans le domaine du tourisme culturel
}

\author{
Experiential marketing and hyperreality in the field of cultural tourism
}

Dominique Bourgeon-Renault et Elodie Jarrier

\section{Introduction}

L'intérêt croissant pour le « patrimoine » coïncide avec le virage postmoderne. Il peut lui aussi être considéré comme une « institution postmoderne » car il reflète l'aspect le plus étudié et le plus

débattu du postmodernisme, le concept

d'hyperréalité.

(Mac Lean, 1997, p. 16)

1 Le caractère hyperréel du patrimoine s'exprime fortement à travers la diversité des formes muséales postmodernes. Après une période de désenchantement du monde, la fête est devenue primordiale dans l'existence des individus (Maffesoli, 1988, 1990). Désormais, les lieux touristiques et culturels proposent une mise en scène "théâtralisée » qui suscite des réactions émotionnelles, ludiques et esthétiques de la part des visiteurs, et implique une appropriation ou une participation de leur part. Dans cette course au « réenchantement» du monde (Ritzer, 1999), le musée n'échappe pas à la règle. Il constitue, au contraire, par l'accent mis sur ses dimensions architecturales, spectaculaires et événementielles, l'un des premiers vecteurs de la «mise en désir d'une ville» (Lefort, 2015, p. 35).

Croiser ville, musée et tourisme renvoie à des enjeux multiples, en particulier d'attractivité, de renouvellement urbain, d'intégration urbaine et d'identité, d'espace public, d'architecture, d'image, et invite à questionner la fonctionnalité du musée dans la ville. [...] Or, si le musée « produit » du tourisme, il n'en est pas moins 
vrai que le tourisme «produit » du musée. [...] De même, si la ville «produit » des musées et du tourisme, réciproquement, le musée et le tourisme " produisent " la ville. (Fagnoni et Gravari-Barbas, 2015, p. 4)

Prenant appui sur ces liens entre tourisme culturel, musées et hyperréalité, cet article mobilise l'approche expérientielle pour décrire et mettre en perspective les trois phases de l'expérience de consommation touristique et culturelle (l'avant expérience, le cœur de l'expérience et l'après expérience). Puis, il explore les stratégies de production d'une expérience touristique et culturelle hyperréelle, décrit ce processus co-créatif et situe la place des dispositifs numériques de médiation dans celui-ci. Enfin, il identifie différentes formes d'immersions hyperréelles spécifiques selon la nature du lieu touristique et culturel appartenant au champ muséal. Ces résultats positionnent le concept d'hyperréalité comme élément de différenciation dans les stratégies élaborées par les structures muséales.

\section{L'expérience touristique et culturelle : un cadre théorique}

3 La consommation touristique et culturelle est une expérience hédonique, multisensorielle, émotionnelle et imaginaire (Holbrook et Hirschman, 1982). L'approche expérientielle analyse la consommation comme un état subjectif primaire provoqué par le symbolisme, l'hédonisme et l'esthétisme du produit. Le tourisme culturel présente tous ces aspects et constitue un terrain particulièrement attirant pour la recherche. Les expériences touristiques et culturelles ont reçu une attention croissante des chercheurs en comportement du consommateur, intéressés par les activités de loisirs en général (Holbrook et Hirschman, 1982), les séjours touristiques (Bonnefoy-Claudet, 2011; Minivielle et Minivielle, 2014), les visites de sites culturels (Petr-Le Huérou, 1998) et/ou les expositions de musées des beaux-arts (Dufresne-Tassé, 1993; Dierking, 1994; Passebois, 2010 ; Jarrier, 2015) ou d'art contemporain (Lagier, 2006 ; De Barnier et Lagier, 2012).

4 Qu'elle soit touristique, culturelle ou muséale, l'expérience vécue peut être expliquée par les caractéristiques intra-individuelles des publics.

\section{Les caractéristiques intra-individuelles explicatives de l'expérience vécue}

5 La perspective expérientielle identifie des caractéristiques intra-personnelles (Bourgeon, 1994 ; Bourgeon et Filser, 1995) de l'individu susceptibles d'expliquer le processus de décision sous-jacent à l'expérience touristique, culturelle ou muséale. Ces caractéristiques individuelles peuvent être présentées ainsi :

- L'implication dans une visite culturelle est une variable importante se situant entre la perception et l'intention d'agir des individus (Bourgeon, 1994 ; Bourgeon et Filser, 1995). L'expérience touristique et culturelle peut susciter des réponses différentes non seulement en termes de niveau d'implication (fort/faible), mais surtout en termes de nature même de l'implication, à dominante cognitive (intérêt) ou à dominante affective (valeur hédonique).

- La recherche de stimulation (Berlyne, 1960 ; Raju, 1980) repose sur l'analyse de l'influence de différentes variables caractérisant le goût de l'individu, d'une part, pour la nouveauté (par 
exemple, transposé au champ touristique, l'attirance pour une destination inhabituelle), d'autre part, pour le changement, et son désir d'acquérir des informations supplémentaires sur les différents produits. De forts niveaux de stimulation sont positivement reliés avec la préférence pour de nouvelles expériences et avec les tendances exploratoires liées aux risques, à la recherche de variété et à la curiosité.

- La tendance à la recherche de sensations (Zuckerman et al.,1964) est susceptible d'expliquer les prédispositions du consommateur à rechercher l'aventure et les frissons, les expériences et la non-inhibition. Dans le domaine touristique et culturel, la perception de l'individu se fonde sur une activité sensorielle qui lui fait vivre une expérience s'exprimant par un simple plaisir ou une intensité de sentiments.

- L'orientation visuelle ou verbale (Childers, Houston et Heckler, 1985; Holbrook, 1986) constituerait une variable explicative des différences de perception de l'objet touristique et culturel. En bref, la tendance à la « visualisation » devrait impliquer une approche holistique de l'objet culturel. Au contraire, la tendance à la « verbalisation » entraînerait une attention plus analytique accordée aux stimuli atomistiques de l'objet.

- La tendance romantique ou classique de la personnalité des individus (Holbrook et Corfman, 1984; Mann, 2000) joue un rôle particulièrement important dans le cadre d'une consommation touristique et culturelle. Le romantisme considère l'expérience de consommation comme une fin en soi et met en avant l'affectif et l'imagination par rapport au cognitif, le dynamisme par rapport au statique et le désordre par rapport à l'ordre. Quant au classicisme, il fait référence à la rationalité, aux valeurs cartésiennes d'ordre, de clarté et de rigueur intellectuelle. Dans une telle perspective, l'individu romantique peut être défini comme un être sensible, créatif, ouvert et peu organisé par opposition à l'individu classique qui est considéré comme étant très organisé, déterminé et se maîtrisant parfaitement.

- Enfin, la nostalgie, que l'on peut définir comme «une réaction affective douce-amère, éventuellement associée à une activité cognitive, [...] éprouvée par un individu lorsqu'un stimulus externe ou interne a pour effet de le transposer dans une période ou dans un événement d'un passé idéalisé, s'inscrivant ou non dans son propre vécu » (Divard et RobertDemontrond, 1997), peut également expliquer le comportement de consommation touristique et culturelle.

6 Après avoir identifié les caractéristiques intra-individuelles susceptibles d'influencer la nature de l'expérience touristique et culturelle, il convient d'en identifier maintenant les composantes saillantes et communes afin de mieux comprendre le vécu des individus.

\section{Les dimensions d'une expérience touristique et culturelle}

7 La littérature a souvent privilégié l'appréhension de l'expérience touristique et culturelle à travers deux de ses dimensions : la dimension sociale et la composante esthétique/ émotionnelle.

8 Tout d'abord, la consommation, en tant qu'expérience sociale, est confirmée par de nombreux auteurs (Maffesoli, 1988, 1990 ; Cova, 1996 ; Rémy, 2000 ; Debenedetti, 2001). Plus particulièrement, l'existence de lien social dans la consommation, valorisée par le courant sociologique postmoderne, a été mise en évidence dans le domaine, d'une part, des concerts de musique classique, à travers la variable « sensibilité à l'interaction sociale » (Mann, 2000), d'autre part, des arts du spectacle vivant, à travers la dimension « lien social » (Pulh, 2002; O'Sullivan, 2009). La consommation culturelle n'est donc pas seulement une expérience individuelle d'ordre esthétique ou intellectuel, c'est aussi une 
expérience sociale qui prend en compte le contexte social intime (Debenedetti, 1998, 1999), composé du spectateur et de ses accompagnateurs. Or, l'individu interagit non seulement avec ses accompagnateurs, mais aussi avec les autres spectateurs et avec tout le personnel en contact, ce qui permet de définir un contexte social élargi (Debenedetti, 1998, 1999).

Ensuite, la participation d'un individu à une visite touristique et culturelle peut être assimilée à une expérience esthétique, proche du paradigme de la réaction émotionnelle. L'émotion apparaît comme un phénomène affectif subjectif expérientiel, source de motivations, qui interagit avec les processus de traitement de l'information recueillie en vue d'une expérience de consommation (Graillot, 1998). Les émotions font partie intégrante de l'expérience vécue (Derbaix et Sjöberg, 1994 ; Richins, 1997).

Cependant, l'expérience touristique et culturelle ne peut pas se réduire uniquement à ces deux composantes (sociale, esthétique/émotionnelle). Comme toute expérience de consommation, elle est susceptible de comporter les quatre dimensions suivantes (Roederer, 2008) :

- une dimension hédonico-sensorielle, qui capte l'intensité de la sensation plaisante procurée par l'expérience à l'individu ;

- une dimension rhétorique, qui exprime les caractéristiques symboliques associées à l'expérience, c'est-à-dire sa fonction de production de sens pour le consommateur ;

- une dimension praxéologique, traduisant le degré d'engagement physique et mental de l'individu dans l'expérience;

- enfin, une dimension temporelle, qui évalue le coût temporel perçu par l'individu pendant l'expérience.

11 Plus spécifiquement, dans le cadre de l'expérience vécue lors d'un séjour touristique en station de ski, Bonnefoy-Claudet (2011) a démontré l'influence positive de la thématisation d'une expérience touristique sur l'émergence d'émotions. Cette thématisation, prenant appui sur des éléments plus ou moins teintés d'hyperréalité, concourt à stimuler et à surprendre l'individu et à lui apporter de la joie ainsi qu'un sentiment de tranquillité. Les recherches de Minivielle et Minivielle (2014) complètent l'identification des dimensions saillantes d'une expérience touristique hyperréelle. Selon ces auteurs, cette expérience peut être appréhendée à travers les six composantes suivantes : sensorielle (stimuli et sensations), émotionnelle (authenticité et découverte), action (exploration et collaboration), tribale (appartenance à un cercle restreint d'initiés), narrative (marqueurs iconographiques et discursifs sur l'imaginaire), et mémorielle (réminiscence, réinterprétation et mise en scène de l'histoire vécue participant de la construction de l'image de soi). Enfin, dans le champ muséal, l'expérience de visite muséale a elle aussi été définie et mesurée à travers six dimensions (Jarrier, 2015) : affective (stimulation, découverte, évasion, fascination, etc.), cognitive et rhétorique (observation, raisonnement, apprentissage, mémorisation, introspection), symbolique (représentations symboliques et métaphoriques, image de soi), sociale (échanges avec les autres visiteurs), spatiale (stratégies de déambulation) et temporelle (gestion de la durée de la visite).

Ce vécu multi-dimensionnel affecte également les comportements et jugements de l'individu après l'expérience. En conséquence, il convient d'identifier les sources de valorisation d'une expérience touristique et culturelle. 


\section{Les sources de valorisation d'une expérience touristique et culturelle}

13 La valeur a longtemps été étudiée en lien avec l'attitude des consommateurs à l'égard du prix, et définie et mesurée de façon duale à travers les notions de bénéfices et de coûts (Day et Crask, 2000). Plus récemment, de nombreuses recherches ont proposé d'étudier la valeur en lien avec l'expérience de consommation, et l'ont définie et mesurée de façon multidimensionnelle (Floch, 1989; Holt, 1995). Ainsi, Holbrook (1994, 1999) définit la valeur comme une préférence relative et interactive caractérisant l'expérience de consommation d'un individu. En conséquence, cette valeur n'est pas unique pour tous les individus, mais possède un caractère multidimensionnel.

L'approche de la valeur de la consommation culturelle se fonde plus spécifiquement sur l'hédonisme, l'esthétisme, le symbolisme et la recherche de lien social dans la relation avec les lieux touristiques et culturels ou encore avec les œuvres d'art. La valeur est alors une réponse affective du consommateur à l'égard de l'objet de consommation. Elle est fonction non pas de l'objet lui-même, mais plutôt de l'expérience de consommation résultant de son utilisation (valeur extrinsèque) ou de son appréciation (valeur intrinsèque). De nombreuses recherches (Aurier, Evrard et N'Goala, 2004 ; Passebois et Aurier, 2004) ont identifié plusieurs dimensions de la valeur selon leur nature expérientielle ou non et selon le caractère intrinsèque/extrinsèque de la consommation culturelle, sur la base d'une distinction entre valeur orientée vers soi et valeur orientée vers les autres. La définition relative et multidimensionnelle a été réaffirmée par Heinich (2017, p. 167) qui propose de définir la valeur comme

la résultante de l'ensemble des opérations par lesquelles une qualité est affectée à un objet, avec des degrés variables de consensualité et de stabilité. Ces opérations sont fonction à la fois de la nature de l'objet évalué, de la nature des sujets évaluateurs et de la nature du contexte d'évaluation.

Dans le champ muséal, Collin-Lachaud et Passebois (2006) ont identifié plusieurs sources de valeurs issues de l'expérience : affective et hédonique, plaisir d'être et de découvrir un lieu, divertissement, esthétique, stimulation expérientielle et sensorielle, variété, surprise et évasion, valeur cognitive, intellectuelle, utilitaire, fonctionnelle, spiritualité, perception mystique d'un lieu, nostalgie, commémoration avec le passé, expression de soi, lien social (ce qui est à relier au plaisir de partager une expérience avec d'autres) et ludique. Plus particulièrement, dans le domaine touristique, Bonnefoy-Claudet (2011) a démontré que la thématisation d'une expérience touristique est source d'enchantement du visiteur et qu'elle contribue à accroître la valeur globale perçue. Enfin, s'intéressant à l'incidence de l'utilisation d'une application mobile sur les sources de valorisation d'une expérience de visite muséale, Nasr, Hallem et Lagier (2017) constatent que les publics peu familiers des smartphones développent leur concentration et leurs connaissances de façon ludique, tandis que les visiteurs habitués aux smartphones accèdent à une réalité dématérialisée, à des compléments d'information qu'ils partagent en temps réel avec leur entourage pour co-promouvoir leur expérience de visite unique et personnalisée. Dès lors, utilisé durant une visite touristique et culturelle, le smartphone peut agir sur différentes sources de la valeur perçue (pédagogique, récréative, sociale, de personnalisation). 
16 Après cette mise en perspective des trois phases de l'expérience de consommation touristique et culturelle (l'avant expérience, le cœur de l'expérience et l'après expérience), il s'avère important de se tourner du côté des professionnels afin d'explorer les stratégies de production d'une expérience touristique et culturelle.

\section{Stratégies de production d'une expérience touristique culturelle hyperréelle}

Du fait des transformations sociétales caractérisant l'ère postmoderne, la place du public se trouve profondément modifiée. Le public est passé d'un statut de "visiteurconsommateur" passif à celui de visiteur acteur de son expérience (Le Brun-Cordier, 2001 ; Le Marec et Deshayes, 1997). Le public est capable de reconstruire le sens des messages qui lui sont transmis et de construire sa propre expérience.

Selon les travaux de recherche du psychologue Csikszentmihalyi (1997), la meilleure expérience (expérience extrême) est celle dite de flux (flow), c'est-à-dire un moment exceptionnel (extraordinaire et inoubliable) pendant lequel « ce que nous sentons, ce que nous souhaitons et ce que nous pensons sont en totale harmonie» (Csikszentmihalyi, 1997).

\section{L'immersion dans l'expérience touristique et culturelle}

19 Le consommateur « fait corps » avec l'expérience durant laquelle toute distance entre lui et le contexte situationnel est annulée.

\section{Un processus co-créatif}

D'une façon synthétique, la production d'expérience peut se faire selon trois aspects (Carù et Cova, 2006) :

- le décor, le design (Bouder Pailler et Damak, 2004) et la mise en scène avec une prise en compte particulière de la stimulation polysensorielle;

- la participation active du consommateur, aidée par les facilitateurs de toute nature, tels que le personnel en contact ou les nouvelles technologies (définissant le rôle de chacun dans les rituels mis en jeu autour de l'offre de l'entreprise) ;

- le récit ou l'histoire qui se noue et qui se prolonge sous la forme, par exemple, de produits dérivés.

21 Les éléments qui définissent la mise en scène d'un événement touristique culturel accentuent la théâtralisation du lieu, avec la définition d'un scénario global propre au lieu ou à l'institution, avec des acteurs « jouant » des rôles précis (le personnel en contact, les visiteurs), avec des décors et éventuellement un fond musical. Ce sont principalement les éléments périphériques à l'offre centrale qui vont participer à la théâtralisation de l'offre globale. Mais le renforcement de la théâtralisation peut concerner également, et surtout, les manifestations touristiques et culturelles elles-mêmes, et pas seulement les services ou éléments périphériques de l'offre globale. Il ne suffit donc pas de déterminer les composantes de la théâtralisation, il convient également de définir son rôle.

Le visiteur peut également être amené à bricoler l'objet culturel, le transformer, le remixer, le commenter, etc. Différents qualificatifs peuvent alors être utilisés pour 
désigner le visiteur : « coproducteur », « cocréateur ", « prosumer » ou encore " pro-am » (professionnel-amateur) (Flichy, 2010). Gamble et Gilmore (2013), à travers de nombreux exemples puisés dans l'industrie musicale, confirment ce paradigme de co-creational marketing, la valeur pouvant être créée par chacun des acteurs (producteur, consommateur).

Par ailleurs, l'association du visiteur, comme co-producteur, peut représenter une condition nécessaire de satisfaction. D'une façon générale, il est possible de favoriser l'appropriation d'une manifestation touristique culturelle par le visiteur lorsque celui-ci est libre de construire lui-même son offre: c'est le cas, par exemple, des Journées du patrimoine.

24 Si l'on prend davantage en compte les services périphériques susceptibles d'accroître la mise en scène de l'espace, et donc de jouer sur la construction de l'expérience vécue, certains éléments apparaissent, qui vont faciliter la participation ou l'appropriation (individuelle ou collective) de l'événement touristique culturel.

\section{La place des dispositifs numériques de médiation culturelle}

À ce propos, dans le domaine muséal, il est important de resituer le rôle important de la médiation, à travers le personnel en contact et les supports écrits ou multimédia, qui peut influencer la valorisation de l'expérience (Chazaud, 1997). Plus spécifiquement, les nouvelles technologies facilitent la médiation entre l'objet culturel et le visiteur en créant de véritables «musées interactifs». Des dispositifs technologiques sont mis en place: audioguides, bornes interactives, écrans tactiles, applications pour mobiles et tablettes... Ils sont qualifiés de self-service technologies ludo-éducatives (edutainment), favorisant la valeur de jeu et présentant une dimension intrinsèque provoquant du "plaisir " (fun) dans l'expérience vécue (Collin-Lachaud et Passebois, 2006 ; Jarrier, 2015). Collin-Lachaud et Passebois (2006) ont cependant mis en garde contre une "sur stimulation » sensorielle, pouvant entraîner des effets pervers (une « MacDonaldisation » de la culture).

Plus récemment, d'autres travaux de recherche (Jarrier et Bourgeon-Renault, 2012 ; De Miguel, Jarrier et Bourgeon-Renault, 2015) ont souligné la complémentarité :

- d'une part, entre les outils interactifs de médiation orientés vers l'apprentissage avec une interactivité assez faible (audioguides, bornes) et ceux qui proposent une expérience plus interactive, divertissante ou multisensorielle (réalité augmentée, etc.), sans occulter cependant leur impact sur la dimension cognitive de la visite ;

- d'autre part, entre ceux qui affectent négativement l'interaction sociale (audioguides, bornes ou smartphone) et ceux qui la stimulent (grandes tables interactives multi-usagers et tablettes tactiles).

\section{La prise en compte de l'hyperréalité dans les stratégies touristiques et culturelles}

Dans le cadre du marketing expérientiel, les professionnels, à travers leur offre touristique et culturelle, peuvent donc amener l'individu à s'approprier l'expérience, 
c'est-à-dire à s'engager dans des processus inoubliables afin de vivre une "suite d'immersions » extraordinaires. et culturelle actuelle est l'hyperréalité (selon laquelle les individus se contentent, pour toute réalité, de celle des images et des copies), il est important de ne pas définir l'immersion seulement comme "un plongeon dans un contexte expérientiel de type Eurodisney " (Carù et Cova, 2006). À la fois état et processus, l'immersion correspond à une perception globale, a posteriori, par les individus de l'état dans lequel ils se trouvaient au cours de l'expérience vécue (Fornerino et al., 2008, p. 101).

31 Afin de comprendre en quoi les univers touristiques et culturels hyperréels sont potentiellement immersifs, il convient de définir le concept d'hyperréalité. Venkatesh et al. (1993) définissent l'hyperréalité comme une construction de la réalité : "une construction des choses plus vraies que la réalité même ». Selon ces auteurs, le réel est à relier à des dimensions de contexte, de culture, d'histoire et d'époque. En conséquence, le concept d'hyperréalité infirme le mythe du réel dans le modernisme. La réalité aurait disparu, laissant place à un monde caractérisé par l'image, l'illusion et la simulation. Selon Graillot (2005a, p. 51), l'hyperréalité peut conduire «à ne plus faire la différence entre "le vrai" et le "faux" ». L'auteur distingue ainsi deux types opposés d'hyperréalité :

- l'une serait fondée sur une copie authentique ou « améliorée » de la réalité matérielle reliant les signifiants à une réalité signifiée ;

- l'autre correspondrait à une copie ne coïncidant pas à un élément original et qui ne reposerait donc pas sur une réalité matérielle; elle peut alors être interprétée comme une réalité originale: «Dans cette perspective, les signifiants sont détachés d'une réalité signifiée » (2005a, p. 51).

Les contours du phénomène d'hyperréalité touristique ont été précisés. Il présente huit principales caractéristiques : une certaine démesure, la thématisation, le rapprochement et/ou la confusion de dimensions hétérogènes et/ou contradictoires, l'intérêt pour le 
concept du temps, pour les détails, la mise en scène de dimensions extrêmes et contrastées, la volonté de contrôler les risques ou sensations déplaisantes ainsi que de générer une immersion grâce à des concepts projectifs parfois régressifs (Graillot, 2005b, p. 6).

Les musées semblent présenter un plus ou moins grand nombre de caractéristiques hyperréelles. Néanmoins, le genre muséal est susceptible d'influencer le recours plus ou moins grand aux stratégies de génération de l'hyperréalité.

\section{Des formes d'immersion hyperréelles spécifiques selon la nature du lieu touristique et culturel}

Graillot (2005a) dénombre quatre stratégies de génération de l'hyperréalité :

- la conception de copies « authentiques » et/ou légèrement « améliorées » d'originaux ;

- la conception de copies « plus réelles » d'originaux;

- la transformation du tout faux en totalement réel ;

- la juxtaposition, voire la confusion, de dimensions hétérogènes et/ou contradictoires.

À partir de cette typologie de stratégies, il paraît intéressant d'analyser comment les professionnels se sont appropriés le concept d'hyperréalité en direction des visiteurs et selon la nature du genre muséal.

Dans ce cadre, nous avons retenu plusieurs genres muséaux en fonction du degré expérientiel de l'offre (de l'éducation, ou dimension cognitive, au divertissement, ou dimension affective de plaisir, d'expérience partagée). En conséquence, nous avons adopté un processus de benchmarking pour étudier et analyser les différentes stratégies de production d'une expérience hyperréelle.

Trois genres muséaux ont ainsi été identifiés: les musées d'art (forte dimension éducative, mais également affective), les musées des sciences ou de techniques (dimension ludico-éducative ou edutainment) et les muséo-parcs archéologiques (forte dimension de divertissement ou entertainment).

\section{Le cas des musées d'art}

Bien que l'on puisse penser que les musées d'art (dont la mission porte sur les collections) ne sont pas concernés par une approche de type Disney consistant «à créer d'abord un imaginaire, qui ne s'inspire pas du consommateur, mais est entièrement conçu par des spécialistes et auquel tous les acteurs [...] pourront adhérer» (Firat et al., 1995), ces derniers semblent toutefois recourir fréquemment aux deux premières stratégies de génération d'hyperréalité.

En effet, il n'est pas rare que les expositions soient thématiques et thématisées plutôt que chronologiques, que l'on propose des regards croisés entre artistes, œuvres ou époques... Il est également possible de retrouver dans ces lieux tant la copie authentique que la copie "améliorée ». Les dispositifs interactifs de médiation, qui incluent de la réalité augmentée, contribuent à exacerber le souci du détail, en permettant, par exemple, de visionner une œuvre d'art et de découvrir diverses restaurations quasi invisibles à l'œil nu grâce à technologies infrarouges ou ultra-violettes. Enfin, au sein des musées américains, la coexistence de reproductions et d'originaux favorise «la falsification». Graillot (2005a, p. 54) confirme ainsi que pour aider le visiteur à faire la différence, les médiateurs installent des panneaux mêlant copies et originaux (Eco, 1985, 1986). 
作, le recours des musées d'art à des éléments hyperréels pose la question de leur perception par les publics. L'expérience artistique est par nature subjective et certains visiteurs valorisent plus que d'autres la rencontre intime et authentique avec une œuvre. En effet, Cova et Cova (2002) identifient deux profils d'individus en quête d'authenticité :

- ceux qui ne recherchent que de l'authenticité « véritable»;

- «ceux qui se contentent d'un ersatz d'authenticité hyperréel apparaissant comme moins risqué et plus édulcoré qu'un produit authentique. » (Graillot, 2005a, p. 51)

41 Ainsi, il semble que la première catégorie d'individus est susceptible de percevoir la présence d'éléments hyperréels comme une entrave à l'appréciation pure de l'œuvre. En effet, on ne peut exclure la possible survenance de comportements de résistance, de détournement ou de réappropriation, témoignant de la volonté des individus de recouvrer leur subjectivité et de subir moins passivement les contraintes de ces environnements hyperréels (Graillot, 2005a, p. 57). En outre, il existerait « un seuil de simulation au-delà duquel les individus rejettent le faux, seuil de simulation parfois dépassé dans les environnements hyperréels » (Graillot, 2005a, p. 58).

\section{Le cas des muséographies immersives dans les musées des sciences ou de techniques}

Si le musée d'art privilégie, encore aujourd'hui, un recours croissant mais modéré aux dispositifs interactifs et immersifs (dans le but de valoriser les œuvres et de favoriser leur appréciation cognitive ou esthétique), d'autres institutions font le choix de recourir à des muséographies immersives (présentations métaphoriques qui conduisent à un déplacement de l'intérêt du contenu vers la forme) (Belaën, 2005, p. 100). En effet, à l'heure actuelle, de nouvelles formes de muséographie se développent à travers des expositions d'immersion qui présentent notamment les caractéristiques suivantes:

- le recours au principe de la simulation afin de créer l'illusion d'une situation, en jouant de l'artifice de type « comme si vous y étiez »;

- l'emphase mise sur la suggestion et non plus sur l'interprétation ;

- l'intégration physique du visiteur dans un environnement clos et fortement scénographié et la volonté de lui faire jouer un rôle actif dans la reconstruction d'un univers de référence en rapport avec la thématique ;

- l'abandon de l'approche pédagogique au profit d'une démarche faisant appel à l'émotion et aux sensations, éléments déclencheurs du désir de connaissance de la part de l'individu (Belaën, 2003, 2005).

Les musées de sciences ou de techniques qui recourent aux expositions d'immersion paraissent donc réunir un plus grand nombre de caractéristiques hyperréelles que les musées d'art. En effet, la thématisation et la scénographie prennent davantage d'ampleur, de même que l'intérêt porté au temps. L'objectif est de donner au visiteur l'impression d'être dans « un temps et un lieu particuliers » (Belaën, 2005, p. 95). Dans certains cas, à défaut de pouvoir pénétrer ces "espaces-temps» dans leur contexte d'origine, ils peuvent être transposés dans les institutions, ou reconstruits (Belaën, 2005, p. 95). Tout semble aller dans le sens d'une nouvelle muséographie «immersive » avec la création de musées virtuels ou de e-galeries à travers le développement d'une interaction accrue.

Toutefois, que les expositions d'immersion soient visitées in situ ou via le site internet de l'institution, le recours à des éléments hyperréels suscite chez le visiteur un panel de réactions diverses et nuancées, rappelant que « l'appropriation de ces dispositifs malgré 
leurs promesses n'est pas immédiate » (Belaën 2003, p. 13). Les travaux de Belaën (2005) insistent sur la tension entre un effet d'immersion et un effet de distanciation, fonction de l'adhésion du visiteur au dispositif. Ils confirment ainsi l'existence de cinq types de réactions possibles face à une muséographie immersive, allant de son appropriation à son rejet de la part du public : la résonance (complète adhésion aux dispositifs), la submersion (individu noyé dans ses émotions par rapport au vécu sans aucun recul), la distanciation critique, la banalisation ou le rejet.

\section{Le cas des muséo-parcs archéologiques}

Outre les musées d'art ou les expositions d'immersions dans les musées de sciences ou de techniques, les muséo-parcs constituent une autre catégorie de structures culturelles qui offrent des univers hyperréels. Ils font partie des parcs de loisirs scientifiques et cherchent à se distinguer des «musées archéologiques traditionnels, qualifiés de lieux poussiéreux, ennuyeux et mortifères » (Roy, 2005, p. 37). Leur recours plus intensif aux stratégies de génération d'hyperréalité transparaît à travers de nombreux éléments :

- l'accentuation des détails et l'emphase mise sur la thématisation ;

- un concept fondateur: "la proposition d'un voyage dans le temps à partir d'un lieu patrimonial (Chaumier, 2003)»(Roy, 2005, p. 37), un temps passé souvent idéalisé et diachronique dans lequel les visiteurs peuvent s'immerger, voire régresser ;

- une volonté de «remplacer les originaux trop fragiles par des fac-similés, de créer d'un environnement de plein air, un "microclimat préhistorique" accompagné d'un parc animalier [...], et de reconstituer des scènes à caractère "ethno-archéologique" » (Roy, 2005, p. 42);

- la perte de lien entre les vestiges archéologiques et leur contexte signifiant, résultant de leur délocalisation au sein du musée (Roy, 2005, p. 38);

- le rôle actif confié au visiteur invité à expérimenter par lui-même ;

- la transformation du tout faux en totalement réel et la juxtaposition, voire la confusion de dimensions hétérogènes et/ou contradictoires, que Serge Chaumier nomme «diversité bariolée » (2005, p. 31).

Ce dernier point est particulièrement prégnant dans les muséo-parcs archéologiques, lieux qui résultent le plus souvent d'une juxtaposition de deux modes de restitution du patrimoine archéologique plutôt que d'une intégration véritable (Roy, 2005, p. 39). Le principal défi est de parvenir à donner de la congruence à l'ensemble. L'auteur semble mettre en garde ces institutions contre certaines dérives. En effet,

certains parcs, que l'on pourrait qualifier «d'attrape-tout", procèdent à des amalgames [...]. Les grottes, gouffres, cavités naturelles, simples curiosités géologiques au titre du relief karstique, sont souvent peuplées de reconstitutions d'hommes préhistoriques, même si aucune trace d'occupation réelle n'y a été décelée ! [...] Un écart maximum se creuse entre l'extrême pauvreté des vestiges [...] et l'exubérance excessive des reconstitutions de scènes préhistoriques animées par des mannequins, sortis d'ateliers spécialisés, destinés à l'origine au cinéma d'épouvante. (Roy, 2005, p. 46)

47 Le parc archéologique se tourne alors vers les nouvelles technologies et les images virtuelles « ou en 3D » pour faire revivre, « de manière réaliste, voire hyperréaliste », les univers archéologiques oubliés (Roy, 2005, p. 51). Ce faisant, il est à la fois populaire (car il répond à la tendance de l'individu à préférer le simulacre au réel) et critiqué par les détenteurs de la culture savante (parce que, conséquence de l'hyperréalité, il privilégie « la reproduction à l'original, la reconstitution au vestige, la ruine à l'"authentique" (Roy, 
2005, p. 57), l'authenticité visuelle à l'authenticité historique et enfin la surface à la profondeur des choses. » (Graillot, 2005a, p. 44, 49)

Au terme de cette brève radiographie du paysage muséal, fondée sur le phénomène d'hyperréalité, il semble possible de positionner les organisations sur un continuum selon leur genre et selon le recours plus ou moins fort aux éléments constitutifs d'un univers hyperréel. Ces résultats peuvent aider les professionnels à élaborer des stratégies fondées sur l'hyperréalité dans un contexte de marketing expérientiel, en direction des visiteurs, dans un but d'élargissement des publics.

Figure 1. Un continuum du recours aux stratégies de génération d'hyperréalité par les institutions de genres muséaux différents (adapté de Graillot, 2005, p. 55)

Réalité matérielle copiée
Réalité originale créée

\begin{tabular}{llll}
\hline $\begin{array}{l}\text { conception de } \\
\text { copies } \\
\text { authentiques }\end{array}$ & $\begin{array}{l}\text { conception de } \\
\text { copies } \\
\text { plus réelles }\end{array}$ & $\begin{array}{l}\text { transformation } \\
\text { du tout faux en } \\
\text { totalement réel }\end{array}$ & $\begin{array}{l}\text { juxtaposition } \\
\text { de dimensions } \\
\text { contradictoires }\end{array}$ \\
\hline musées d'art & expositions d'immersion & muséo-parcs archéologiques \\
\hline
\end{tabular}

Importance de la subjectivité projetée dans la (re)construction

\section{Conclusion}

Toute expérience touristique et culturelle peut être appréhendée selon trois phases. Avant l'expérience, les caractéristiques intra-individuelles (implication, recherche de stimulation sensorielle, recherche de sensations, orientation visuelle ou verbale, classique ou romantique) interviennent dans le processus de décision de visiter un lieu touristique et culturel. Au cœur de l'expérience touristique et culturelle, de multiples dimensions (sensorielle, émotionnelle, active, tribale, narrative et mémorielle) interagissent (Minivielle et Minivielle, 2014). Après l'expérience, de nombreuses sources de valorisation sont identifiées et présentent de nombreuses similitudes, qu'elles soient issues d'une visite touristique, culturelle ou muséale (Bonnefoy-Claudet, 2011; Collin-Lachaud et Passebois) et renforcées par des outils mobiles de médiation (Nasr, Hallem et Lagier, 2017). Dès lors, dans un souci d'enrichir, voire de réenchanter l'expérience vécue, les professionnels du tourisme culturel mettent en œuvre des stratégies de production d'expérience plus ou moins fortement co-créées, interactives, thématisées ou immersives, voire hyperréelles. Les formes muséales contemporaines illustrent la diversité des immersions hyperréelles produites.

Cependant, certains individus peuvent encore rencontrer des difficultés à déchiffrer le langage des formes, de la mise en espace et de la scénographie. Aussi, convient-il de ne pas négliger l'importance du personnel en contact dans la construction et l'organisation de l'expérience vécue (accueil, médiation, information et communication).

51 Sur un autre plan, le développement des nouvelles technologies peut provoquer, chez le public, une "sur stimulation" sensorielle entraînant des effets pervers. Les structures touristiques et culturelles, favorisant leur accessibilité, sont accusées de désacralisation de leur identité et de «Disneyisation » (ou encore de «MacDonaldisation »), c'est-à-dire 
de « marchandisation de la culture ». Mais ne pourrait-on pas davantage interpréter cette évolution comme l'effacement de la distinction entre culture classique (culture savante) et culture de masse, ce qui semble correspondre à ce que Lahire (2004) nomme le décloisonnement des genres culturels? Sur un plan managérial, cette recherche contribue à réfléchir au positionnement des organisations et, en conséquence, à des éléments de différenciation à introduire dans l'élaboration de leur future stratégie afin de permettre un meilleur accès à leur offre, favorisant ainsi un élargissement de leurs publics.

\section{BIBLIOGRAPHIE}

Florence BELAEN, «L'immersion dans les musées de science : médiation ou séduction? », Revue Culture et Musées, $n^{\circ}$ 5, 2005.

Florence BELAEN, «L'analyse de l'apparition d'un nouveau genre culturel dans les musées des sciences : les expositions d'immersion », $\mathrm{X}^{\text {ème }}$ colloque bilatéral franco-roumain, CIFSIC, Université de Bucarest, Actes électroniques, 2003.

Imed Ben NASR, Yousra HALLEM et Joëlle LAGIER, « Quel est le rôle de l'application mobile dans la valorisation de l'expérience muséale ? ", Management \& Avenir, $n^{\circ}$ 2, 2017.

Daniel Ellis BerLyne, Conflict, Arousal and Curiosity, Mc Graw-Hill Book Company, 1960.

Lydie BONNEFOY-CLAUDET, Les effets de la thématisation du lieu sur l'expérience vécue par le consommateur. Une double approche cognitive et expérientielle, thèse de doctorat en sciences de gestion, Université de Grenoble, 2011.

Danielle BOUDER PAILLER et Leïla DAMAK, « Le design de l'espace de représentation d'un spectacle vivant influence-t-il les attitudes et comportements des spectateurs ? Approche exploratoire ", Actes du colloque sur le design, Nantes, 2004.

Dominique BOURGEON, Essai de modélisation du comportement dans le domaine culturel : une application au secteur théâtral, thèse de doctorat en sciences de gestion, Université de Bourgogne, 1994.

Dominique BOURGEON et Marc FILSER, « Les apports du modèle expérientiel à l'analyse du comportement dans le domaine culturel : une exploration conceptuelle et méthodologique ", Recherches et applications en marketing, vol. 10, n 4, 1995.

Antonella CARÙ et Bernard COVA, « Expériences de consommation et marketing expérientiel ", Revue française de gestion, vol. 32, $\mathrm{n}^{\circ}$ 162, 2006.

Antonella CARÙ et Bernard COVA, « Approche empirique de l'immersion dans l'expérience de consommation : les opérations d'appropriation ", Recherche et applications en marketing, vol. 18, n $\circ 2,2003$.

Anis CHARFI et Pierre VOLLE, "L'immersion dans les environnements expérientiels en ligne : rôle des dispositifs de la réalité virtuelle ", actes du congrès international de l'Association française du marketing, Le Mans, 2010. 
Pierre CHAZAUD, « Marketing de la visite culturelle et implication du public », Revue publics et musées, $\mathrm{n}^{\circ} 11-12,1997$.

Isabelle COLLIN-LACHAUD et Juliette PASSEBOIS, « Le rôle des NTIC dans la valorisation des expériences culturelles et la fidélisation des visiteurs : le cas du Paléosite », $11^{\text {èmes }}$ Journées de recherche en marketing de Bourgogne, Université de Bourgogne, 2006.

Terry L. CHILDERS, Michael J. HOUSTON et Susan E. HECKLER, « Measurement of Individual Differences in Visual Versus Verbal Information Processing ", Journal of Consumer Research, vol. $12, \mathrm{n}^{\circ} 2,1985$.

Bernard CovA, « Leçons de marketing postmoderne », L'Expansion Management Review, 1996.

Véronique cova et Bernard cova, « Les particules expérientielles de la quête d'authenticité du consommateur ", Décisions marketing, $\mathrm{n}^{\circ}$ 8, 2002.

Mihaly CSIKSZENTMIHALYI, Finding Flow, Perseus Book, 1997.

Ellen DAY et Melvin R. CRASK, « Value assessment: the antecedent of customer satisfaction », Journal of Consumer Satisfaction Dissatisfaction and Complaining Behavior, vol. 13, n 1, 2000.

Virginie DE BARNIER et Joëlle LAGIER, « La résistance à l'art contemporain : ses attitudes et représentations des publics aux implications marketing ", Décisions marketing, n 68, 2012.

Marta DE MIGUel DE BLAS, Dominique BoURGeOn-RENAUlt et Élodie JARRIER, « Can Interactive Mediation Tools Bridge the Identity Gap Between the Public and the Art Museum? », International Journal of Arts Management, vol. 18, nº 1, 2015.

Stéphane DEBENEDETTI, Rôle et impact de l'accompagnement du visiteur du lieu culturel. Le cas de la sortie au musée d'art, thèse de doctorat en sciences de gestion, Université de Paris 9 Dauphine, 2001.

Stéphane DEBENEDETTI, « Le contexte social : un facteur déterminant des comportements de sortie culturelle », actes de la $5^{\text {ème }}$ Conférence internationale sur le management des arts et de la culture, Helsinki, 1999.

Stéphane DEBENEDETTI, « La convivialité de groupe dans les sorties culturelles : état de l'art et voies de recherche », actes du $14{ }^{\text {ème }}$ Congrès international de l'Association française $\mathrm{du}$ marketing, 1998.

Christian DERBAIX et Lennart SJÖBERG, « Movie stars in space: A comparison of preference and similarity judgments ", International Journal of Research in Marketing, vol. 11, n 3, 1994.

Lynn Diane DIERKING, « Du musée au parc d'attractions. Rôle de l'interaction sociale dans l'expérience muséale », Revue publics et musées, n 5, 1994.

Ronan DIVARD et Philippe ROBERT-DEMONTROND, « La nostalgie : un thème récent dans la recherche en marketing ", Recherche et application en marketing, vol. 12, n 4, 1997.

Colette DUFRESNE-TASSÉ, « Limites des études sur le comportement du visiteur, potentiel de la recherche sur son fonctionnement psychologique », actes de la $2^{\text {ème }}$ Conférence internationale sur le management des arts et de la culture, Montréal, 1993.

Yves EVRARD, Philippe AURIER et Gilles N'GOALA, « Comprendre et mesurer la valeur du point de vue du consommateur », Recherche et applications en marketing, vol. 19, n 3, 2004.

Edith FAGNONI et Marie GRAVARI-BARBAS, Nouveaux musées, nouvelles ères urbaines, nouvelles pratiques touristiques, coll. « Géographie », Presses de l'Université Laval, 2015.

Nathalie HEINICH, Des valeurs. Une approche sociologique, Gallimard, 2017. 
A. Fuat FIRAT, Nikhilesh DHOLAKIA et Alladi VeNKATESH, « Marketing in a Postmodern World », European Journal of Marketing, vol. 29, n 1, 1995.

Patrice FLICHY, Le sacre de l'amateur. Sociologie des passions ordinaires à l'ère numérique, Seuil-La République des idées, 2010.

Jean-Marie $\mathrm{FLOCH}$, « La contribution d'une sémiotique structurale à la conception d'un hypermarché ", Recherche et applications en marketing, vol. 4, n² 2, 1989.

Marianela FORNERINO, Agnès HELME-GUIZON et Davis GOTTELAND, « Expériences cinématographiques en état d'immersion : effet sur la satisfaction ", Recherche et applications en marketing, vol. $23, \mathrm{n}^{\circ} 3$, 2008.

Jordan GAMBLE et Audrey GILMORE, «A New Era of Consumer Marketing? An Application of Cocreational Marketing in the Music Industry ", European Journal of Marketing, vol. 47, $n^{\circ} 11 / 12$, 2013.

Laurence GRAILLOT, « Réalités (ou apparences?) de l'hyperréalité : une application au cas du tourisme de loisirs ", Recherche et applications en marketing, vol. 20, n 1, 2005a.

Laurence GRAILLOT, « Tentative de caractérisation du phénomène d'hyperréalité touristique : un état de l'art ", actes de la 1 ère journée thématique AFM de recherche en marketing du tourisme et des loisirs, Chambéry, 2005b.

Laurence GRAILLOT, «Émotions et comportement du consommateur ", Recherche et applications en marketing, vol. 13, $\mathrm{n}^{\circ} 1,1998$.

Patrick HETZEL, Planète conso : marketing expérientiel et nouveaux univers de consommation, Éditions d'organisation, Paris, 2002.

Morris B. HOLBROоK, Consumer Value: A Framework for Analysis and Research, Routledge, 1999.

Morris B. HоLвRоок, « The Nature Of Customer Value: An Axiology of Services In The Consumption Experience, Service Quality », dans Roland T. RUST et Richard L. OLIVER (dir.), Service Quality: New Directions in Theory and Practices, Sage Publications, 1994.

Morris B. HOLBROOK, « Aims, Concepts et Methods for the Representation of Individual Differences in Esthetic Responses to Design Features », Journal of Consumer Research, vol. 13, n 3, 1986.

Morris B. HOLBROOK et Kim P. CORFMAN, « Quality and Value in the Consumption Experience: Phaedrus Rides Again ", dans Jacob JACOBY et Jerry oLson (dir.), Perceived Quality: How Consumers View Stores and Merchandise, Lexington Books, 1984.

Morris B. HOLBROOK et Elizabeth C. HIRSCHMAN, « The Experiential Aspects of Consumption: Consumer Fantasies, Feelings and Fun », Journal of Consumer Research, n 9, 1982.

Douglas B. HOLT, « How consumers consume: A typology of consumption practices ", Journal of Consumer Research, vol. 22, n 1, 1995.

Élodie JARRIER, Une approche expérientielle des effets de l'utilisation d'un outil interactif dans le domaine culturel : une application aux musées d'art, thèse de doctorat en sciences de gestion, Université de Bourgogne, 2015.

Élodie JARRIER et Dominique BOURGEON-RENAULT, «Impact of Mediation Devices on the Museum Visit Experience and on Visitors' Behavioural Intentions », International Journal of Arts Management , vol. 15, n 1, 2012.

Joëlle LAGIER, Le style esthétique, caractéristique individuelle de la perception et du jugement à l'égard de l'objet, thèse de doctorat en sciences de gestion, Université de Bourgogne, 2006. 
Bernard LAHIRE, La culture des individus, dissonances culturelles et distinction de soi, La Découverte, 2004.

Pascal LE BRUN-CORDIER, Renforcer le lien entre l'étude des publics et l'action culturelle, Les institutions culturelles au plus près du public, La Documentation française, 2001.

Joëlle LE MAREC et Stéphanie DESHAYES, «Évaluation, marketing et muséologie », Revue publics et musées, n 11-12, 1997.

Isabelle LEFORT, « L'identité lyonnaise au risque de ses musées », dans Edith FAGNONI et Marie GRAVARI-BARBAS (dir.), Nouveaux musées, nouvelles ères urbaines, nouvelles pratiques touristiques, coll. « Géographie », Presses de l'Université Laval, 2015.

Fiona MACLEAN, « Le passé est à vendre. Réflexions sur le marketing des musées », Revue publics et musées, vol. 11-12, 1997.

Michel MAfFesoli, Au creux des apparences. Pour une éthique de l'esthétique, Plon, 1990.

Michel MAFFESOLI, Le temps des tribus : le déclin de l'individualisme dans les sociétés de masse, Méridiens-Klincksieck, 1988.

Patricia MANN, La sensibilité esthétique et la sensibilité à l'interaction sociale : deux nouvelles variables pour expliquer le comportement de fréquentation des concerts de musique classique, thèse de doctorat en sciences de gestion, Université de Bourgogne, 2000.

Terry o'sulLIVAN, « All together now: A symphony orchestra audience as a consuming community », Consumption, Markets and Culture, vol. 12, n 3, 2009.

Juliette PASSEBOIS, « Bâtir et entretenir une relation avec les visiteurs d'une institution artistique ", dans Isabelle ASSASSI, Dominique BOURGEON-RENAULT et Marc FILSER (dir.), Recherches en marketing des activités culturelles, 2010.

Juliette PASSEBOIS et Philippe AURIER, « Le rôle de l'expertise des consommateurs dans l'expérience culturelle : une approche par la valeur de consommation ", $9^{\text {èmes }}$ Journées de recherche en marketing de Bourgogne, 2004.

Christine PETR-LE HUÉROU, Le phénomène de fréquentation touristique du patrimoine en vue de sa gestion : le cas des sites mégalithiques du Morbihan, thèse de doctorat en sciences de gestion, Université de Rennes 1, 1998.

B. Joseph PINE et James H. GILMORE, The Experience Economy: Work is a Theatre and Every Business is a Stage, HBS Press, 1999.

Mathilde PULH, La valorisation de l'expérience de consommation d'activités culturelles : le cas des festivals d'arts de la rue, thèse de doctorat en sciences de gestion, Université de Bourgogne, 2002.

Puthankurissi S. RAJU, «Optimal Stimulation Level: its Relationship to Personality, Demographics, and Exploratory Behavior ", Journal of Consumer Research, vol. 7, n 3, 1980.

Éric REMY, Le lien social dans les échanges marchands de service : concept de services de lien et habillage social, thèse de doctorat en sciences de gestion, Université de Rouen, 2000.

Marsha L. RICHINS, " Measuring emotions in the consumption experience », Journal of Consumer Research, vol. 24, n², 1997.

George RITZER, Enchanting a Disenchanted World. Revolutionizing the Means of Consumption, Pine Forge Press, 1999. 
Claire ROEDERER, L'expérience de consommation : exploration conceptuelle, méthodologique et stratégique, thèse de doctorat en sciences de gestion, Université de Bourgogne, 2008.

Jean-Bernard RoY, «Les parcs archéologiques au risque du parc de divertissement : essai d'approche typologique ", Revue culture et musées, n 5, 2005.

Alladi VENKATESH, John F. SHERRY Jr et A. Fuat FIRAT, « Postmodernism and the Marketing Imaginary », International Journal of Research in Marketing, vol. 10, n 3, 1993.

Marvin ZUCKERMAN, Elizabeth A. KOLIN, Leah PRICE et Ina ZOOB, « Development of a sensationseeking scale ", Journal of Consulting Psychology, vol. 28, n 6, 1964.

\section{RÉSUMÉS}

Le caractère hyperréel du patrimoine s'exprime fortement à travers la diversité des formes muséales postmodernes. Prenant appui sur les liens existant entre le tourisme culturel, le domaine muséal et le concept d'hyperréalité, cet article mobilise l'approche expérientielle tout d'abord pour décrire et mettre en perspective les trois phases de l'expérience de consommation touristique et culturelle (l'avant expérience, le cœur de l'expérience et l'après expérience). Puis, il explore les stratégies de production d'une expérience touristique et culturelle hyperréelle, décrit ce processus co-créatif et situe la place des dispositifs numériques de médiation dans celuici. Pour terminer, il identifie différentes formes d'immersions hyperréelles spécifiques en fonction de la nature du lieu touristique et culturel permettant d'observer les effets de ces stratégies sur l'expérience de visite des musées.

The hyperreal character of the heritage is strongly expressed through the diversity of postmodern museum forms. Drawing on the links existing between cultural tourism, the museum domain and the concept of hyperreality, this article mobilizes the experiential approach first to describe and emphasize the three phases of the tourism and cultural consumption experience (the pre-experience, the core of the experience and the post-experience). Then, it explores the strategies of producing a hyperreal tourist and cultural experience, describes this co-creative process, and situates digital mediation devices in it. Finally, it identifies different forms of specific hyperreal immersions depending on the nature of the tourist and cultural place, allowing to observe the effects of these strategies on the museum experience.

\section{INDEX}

Keywords : experiential marketing, cultural tourism, lived experience, immersion, hyperreality Mots-clés : marketing expérientiel, tourisme culturel, expérience vécue, immersion, hyperréalité

\section{AUTEURS}

\section{DOMINIQUE BOURGEON-RENAULT}

Professeur des Universités en marketing

CREGO EA 7317

Université de Bourgogne

2, Bd. Gabriel 
BP 26611

21066 DIJON Cedex

dominique.renault-bourgeon@orange.fr

\section{ELODIE JARRIER}

Maître de conférences en marketing

GRANEM EA 7456

Université d'Angers

13, Allée François Mitterrand

elodie.jarrier@univ-angers.fr 